\title{
Inter- and Intramolecular Interactions of Polymer as Studied by Fluorescence Spectroscopy XV. Solvent and Concentration Effects on the Exciplex Emission by Poly[oxy-2,2-(1-pyrenylmethyl) (4-N,N-dimethylaminobenzyl)propyleneoxysebacoyl]
}

\author{
Hui Liang YUAN and Shigeo TAZUKE \\ Research Laboratory of Resources Utilization, \\ Tokyo Institute of Technology, \\ 4259 Nagatsuta, Midori-ku, Yokohama, Japan
}

(Received July 5, 1982)

\begin{abstract}
Poly[oxy-2,2-(1-pyrenylmethyl)(4- $N, N$-dimethylaminobenzyl)propyleneoxysebacoyl] (III) (DP (degree of polymerization) \$38) and its reference polymer, poly[oxy-2,2-(1pyrenylmethyl)(benzyl)propyleneoxysebacoyl] (V) were prepared by polycondensation of the corresponding 1,3-propanediol with thiophenyl sebacate. The effects of solvents and the concentration on the inter- and intramolecular exciplex formation by III were examined in three solvents and compared with the monomer model compounds, 1,3-Diacetoxy-2-(4- $N, N$ dimethylaminobenzyl)-2-(1-pyrenylmethyl)propane (IV) and 1,3-diacetoxy-2-benzyl-2-(1pyrenylmethyl)propane (VI). The ratio of the exciplex emission intensity $\left(F_{\mathrm{e}}\right)$ to the monomer emission intensity of pyrenyl groups $\left(F_{\mathrm{m}}\right)$ increased with concentration in a region below $10^{-5} \mathrm{M}$, suggesting the interpolymer exciplex formation of III, whereas the exciplex by IV was concentration independent. The degree of interpolymer exciplex formation was higher in 1,2-dichloroethane (good solvent) than in mesitylene or $o$-xylene (poor solvent). The optimum DP for interpolymer exciplex formation was observed. On the other hand, the intrapolymer exciplex intensity $\left(\left(F_{\mathrm{e}} / F_{\mathrm{m}}\right)_{c \rightarrow 0}\right)$ of III increased monotonously with DP, suggesting non-neighboring group participation in the intrapolymer exciplex formation, since the excimer formation by $\mathbf{V}$ is entirely an intrapolymer event and VI emits fluorescence from the locally excited state of pyrene alone. The driving force for the intermolecular association in the polymeric systems was attributed to the weak ground state interactions between the electron donor ( $N, N$-dimethylanilino group, DMA) and the electron acceptor (pyrenyl group, Py). The quantum yield of the exciplex emission by III in 1,2dichloroethane was as high as 0.4 . The factors determining the interpolymer association are also qualitatively discussed.
\end{abstract}

KEY WORDS Polyester / Fluorescence / Exciplex / Electron Donor Acceptor Complex / Concentration Effect / Solvent Effect / Molecular Weight Effect / Polymer Association / Pyrene / N,N-Dimethylaniline /

The emission behavior of polymer bound exciplexes has shown to provide useful information on intra- and interpolymer chromophore interactions both in excited and ground states, environmental effects on exciplex emission, segment mobility of polymer chains and so forth. Among the exciplex forming pairs so far studied, we chose pyrenyl(Py) and $N, N$-dimethylanilino (DMA) groups as an acceptor and a donor, respectively, and carried out spectroscopic measurements with a polyester in which Py and DMA were arranged in an alternate fashion (I $)^{1-4}$ and a polymethacrylate having pendant 1-(1-pyrenyl)-3-( $N, N$-dimethylanilino)propane groups (II) ${ }^{5,6}$ Our findings agreed well with our previous results on the polymer bonded exciplexes of the carbazole-terephthalate or anthracene-DMA pair. ${ }^{7-14}$ Although we studied the effects of molecular weight, concentration and solvent on the exciplex emission, the drawbacks of working with I are the difficulty of preparing high molecular weight 
polymers and the fixed choice of the main chain structure.

Recently, we developed a new synthetic process for preparing high molecular weight polyesters starting from 2,2-disubstituted-1,3-propanediols which are hopelessly inactive in the conventional polyester synthesis by the ester exchange method. ${ }^{22}$ This process has enabled us to investigate a series of polyesters having the general structure of poly[oxy2,2-disubstituted-propyleneoxycarbonylalkylenecarbonyl]. The advantage of these samples is the wide range of choice of chromophore interval and molecular weight. Furthermore, the diol monomer can be converted to polyurethanes of comparable structure..$^{15-17}$

First, we prepared samples III-VI. The exciplex forming polymer III is different from I in the following respects; i) Py and DMA are connected by three methylene groups so that the $n=3$ rule $^{18}$ is satisfied. Consequently, III resembles II. ii) The interval between the exciplex forming pairs is very large so that the behavior of intra- and interpolymer chromophore association may be quite different from that of I and II. The exciplex foming monomer model (IV) is used to examine the intramolecular exciplex formation between adjacent chromophores. The polymer $\mathbf{V}$ is a model polymer which does not form exciplex. The excimer formation between Py groups in which the phenyl group does not participate is compared with the exciplex formation by III. ${ }^{19}$ VI is a monomer model for $\mathrm{V}$ and exhibits emission only from the locally excited state of Py. Using these samples the characteristics of III are discussed in comparison with I and II.

\section{EXPERIMENTAL}

\section{Materials}

Poly[oxy-2,2-(1-pyrenylmethyl) (4-N,N-dimethylaminobenzyl)propyleneoxysebacoyl] (III). The monomer, 2-(4- $N, N$-dimethylaminobenzyl)-2-(1pyrenylmethyl)propane-1,3-diol, was prepared from diethyl 2-(4- $N, N$-dimethylaminobenzyl)-2(1-pyrenylmethyl) malonate ${ }^{5}$ by reduction with $\mathrm{LiAlH}_{4}$ in dry tetrahydrofuran. Yield $50.1 \%$; mp $167-168^{\circ} \mathrm{C}$, IR ( $\mathrm{KBr}$ disk) $3300 \mathrm{~cm}^{-1}\left(v_{\mathrm{OH}}\right)$. Analytical data: E.A. Calcd for $\mathrm{C}_{29} \mathrm{H}_{29} \mathrm{O}_{2} \mathrm{~N}: \mathrm{C}$, $82.28 \%$; H, 6.85\%; N, 3.30\%. Found: C, $82.50 \%$; $\mathrm{H}, 7.03 \%$; N, 3.31\%. NMR $\left(\mathrm{CDCl}_{3}\right) \delta 2.75(2 \mathrm{H}$, s, $\left.-\mathrm{CH}_{2}-\mathrm{Ph}\right), \delta 2.90\left(6 \mathrm{H}, \mathrm{s},-\mathrm{N}\left(\mathrm{CH}_{3}\right)_{2}\right), \delta 3.49(2 \mathrm{H}$, s, - $\left.\mathrm{CH}_{2}-\mathrm{Py}\right), \delta 3.51-3.65\left(4 \mathrm{H}, \mathrm{d},>\mathrm{C}\left(\mathrm{CH}_{2} \mathrm{OH}\right)_{2}\right)$, $\delta 2.05-2.22(2 \mathrm{H}, \mathrm{t},-\mathrm{OH}), \delta 6.57-7.15(4 \mathrm{H}, \mathrm{m}$, -phenyl), and $\delta 7.90-8.23$ (9H, m, -Py).

The polymer was prepared by the method already reported. ${ }^{20,21}$ The fractionation and molecular weight measurement of the polymers were made by GPC (HLC-802UR, Toyo Soda Mfg. Co. Ltd; fractionation column, $\mathrm{G} 2000 \mathrm{HG}_{6}+\mathrm{G} 3000 \mathrm{HG}_{6}$, analytical column; $\mathrm{GMH} \times 2+\mathrm{G} 4000 \mathrm{H}+$ $\mathrm{G} 2500 \mathrm{H}$, eluent : chloroform). E.A. Calcd for $\mathrm{C}_{39} \mathrm{H}_{43} \mathrm{O}_{4} \mathrm{~N}$ (repeating unit): $\mathrm{C}, 79.43 \% ; \mathrm{H}, 7.35 \%$; $\mathrm{N}, 2.37 \%$. Found: C, $80.05 \%$; H, $7.20 \%$; $2.19 \%$.

Poly[oxy-2,2-(1-pyrenylmethyl) (benzyl)propyleneoxy sebacoyl (V). The monomer of 2-(1-pyrenylmethyl)-2-(benzyl)propane-1,3-diol was prepared from diethyl 2-(1-pyrenylmethyl)-2-(benzyl) malonate by the same method mentioned above. Yield $90.1 \%$; mp 50.5-51.0 ${ }^{\circ} \mathrm{C}$; IR ( $\mathrm{KBr}$ disk) $3400 \mathrm{~cm}^{-1}$ $\left(v_{\mathrm{OH}}\right)$. Analytical data: E.A. Calcd for $\mathrm{C}_{27} \mathrm{H}_{24} \mathrm{O}_{2}$ : C, $85.23 \%$; $\mathrm{H}, 6.36 \%$. Found: $\mathrm{C}, 85.15 \%$; $\mathrm{H}, 6.30 \%$. NMR $\left(\mathrm{CDCl}_{3}\right) \delta 1.98(2 \mathrm{H}, \mathrm{s},-\mathrm{OH}), \delta 2.85(2 \mathrm{H}, \mathrm{s}$, $\left.-\mathrm{CH}_{2}-\mathrm{Ar}\right), \delta 3.40\left(2 \mathrm{H}, \mathrm{s},-\mathrm{CH}_{2}-\mathrm{Py}\right), \delta 3.60(4 \mathrm{H}, \mathrm{s}$, $\left.-\mathrm{CH}_{2} \mathrm{OH} \times 2\right), \delta 7.40(5 \mathrm{H}, \mathrm{s}$, phenyl), and $\delta 7.90$ $8.35(9 \mathrm{H}, \mathrm{m},-\mathrm{Py})$.

The polymer (V) was prepared by the same method as reported for III. The molecular weight was estimated by GPC analysis. Analytical data: E.A. Calcd for $\mathrm{C}_{37} \mathrm{H}_{38} \mathrm{O}_{4}$ (repeating unit): C, $81.40 \% ; \mathrm{H}, 6.96 \%$. Found: C, $80.95 \% ; \mathrm{H}, 6.90 \%$.

1,3-Diacetoxy-2-(4- $N, N$-dimethylaminobenzyl)2-(1-pyrenylmethyl)propane (IV). IV was prepared from 2-(4- $N, N$-dimethylaminobenzyl)- 2-(3pyrenylmethyl) propane-1,3-diol and acetyl chloride in benzene in the presence of triethylamine as a catalyst. The product was purified on an alumina gel column. Yield $69.5 \%$; mp 59-60 $0^{\circ} \mathrm{C}$. IR $(\mathrm{KBr}$ disk) $1720 \mathrm{~cm}^{-1}\left(v_{\mathrm{C}=0}\right)$. Analytical data: E. A. Calcd for $\mathrm{C}_{33} \mathrm{H}_{33} \mathrm{O}_{4} \mathrm{~N}$ : C, $78.08 \% ; \mathrm{H}, 6.55 \%$; N, $2.76 \%$. Found: C, $77.92 \%$; H, 6.59\%; N, $2.62 \%$. $\operatorname{NMR}\left(\mathrm{CDCl}_{3}\right) 2.00\left(6 \mathrm{H}, \mathrm{s},-\mathrm{COCH}_{3}\right), \delta 2.85(2 \mathrm{H}, \mathrm{s}$, $-\mathrm{CH}_{2}$-phenyl), $\delta 2.90\left(6 \mathrm{H}, \mathrm{s},-\mathrm{N}\left(\mathrm{CH}_{3}\right)_{2}\right), \delta 3.57(2 \mathrm{H}$, $\left.\mathrm{s},-\mathrm{CH}_{2}-\mathrm{Py}\right), \delta 3.96\left(4 \mathrm{H}, \mathrm{s},-\mathrm{OCOCH}_{2}-\times 2\right), \delta 6.66$ $7.49(4 \mathrm{H}, \mathrm{m}$, phenyl), and $\delta 7.70-8.33(9 \mathrm{H}, \mathrm{m}, \mathrm{Py})$.

1,3-Diacetoxy-2-benzyl-2-(1-pyrenylmethyl) propane (VI). VI was prepared by the same method described above. Yield 48.5\%. IR (KBr disk) 1710 $\mathrm{cm}^{-1}\left(v_{\mathrm{C}=0}\right)$. Analytical data: E.A. Calcd for $\mathrm{C}_{31} \mathrm{H}_{28} \mathrm{O}_{4}$ : C, $80.15 \% ; \mathrm{H}, 6.07 \%$. Found: $\mathrm{C}$, $80.10 \% ; \mathrm{H}, 6.05 \%$. NMR $\left(\mathrm{CDCl}_{3}\right) \delta 1.98(6 \mathrm{H}, \mathrm{s}$, $\left.-\mathrm{COCH}_{3}\right), \delta 2.85\left(2 \mathrm{H}, \mathrm{s},-\mathrm{CH}_{2}\right.$-phenyl), $\delta 3.45(2 \mathrm{H}$, 
s, -CH-Py), $\delta 3.85\left(4 \mathrm{H}, \mathrm{s},-\mathrm{OCOCH}_{2}-\times 2\right), \delta 7.25$ $(5 \mathrm{H}, \mathrm{s}$, phenyl), and $\delta 7.85-8.40(9 \mathrm{H}, \mathrm{m}, \mathrm{Py})$.

\section{Spectroscopy}

Absorption and fluorescence spectra were recorded on a Shimadzu UV-200 spectrometer and a Hitachi MPF-4 spectrofluorometer, respectively. For fluorescence spectroscopy, sample solutions were purged with argon to eliminate air and excited at $346 \mathrm{~nm}$. The slit width was maintained at $3 \mathrm{~nm}$ on either side of the emission and excitation. All the emission spectra were not corrected. The molarity of the polymer solution was based on the "repeating unit" concentration. The exciplex emission quantum yield $\left(\Phi_{\mathrm{e}}\right)$ was measured as a function of concentration as reported ${ }^{22}$ already.

\section{Solvent}

Mesitylene was twice distilled before use. Spectrograde $o$-xylene and 1,2-dichloroethane (DCE) were further distilled before use. Other solvents were purified by the usual methods.

\section{RESULTS}

\section{Absorption Spectra}

The absorption spectra of samples III, IV, $\mathbf{V}$, and VI in the following scheme are shown in Figure 1. I and II were reported previously and cited again for comparison. In the $S_{0}-S_{2}$ absorption band, the

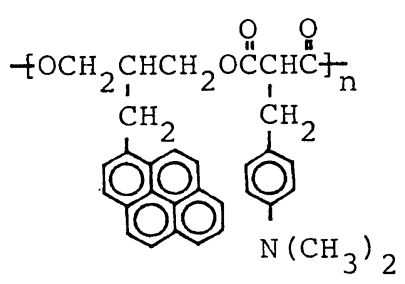

I

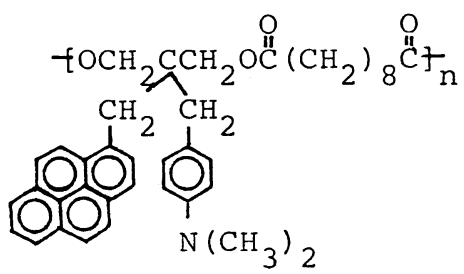

III

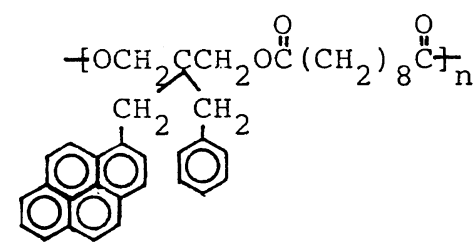

V

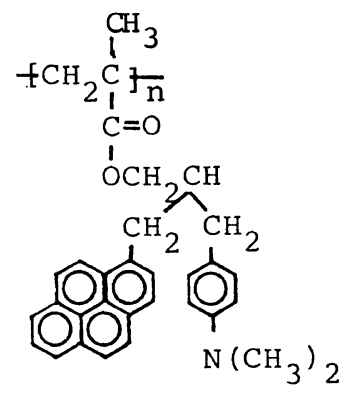

II

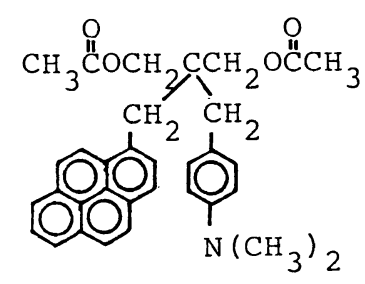

IV

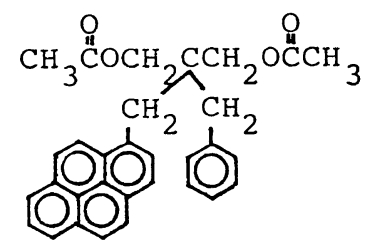

VI

Scheme 1. Sample structures. 
absorption spectrum of III showed a red shift of 2$3 \mathrm{~nm}$ relative to $\mathbf{I V}$ and $\mathbf{V}$. Although the qualitative absorption profiles are identical for III and IV or V, the spectrum of III is broader than that of IV and V, in particular above $350 \mathrm{~nm}$. As a measure of broadness, the ratio of maximum absorbance at $335 \mathrm{~nm}$ (valley) to the minimum absorbance at $346 \mathrm{~nm}$ (peak) was calculated as shown in Table I. The broadness of absorption spectra of III was found to depend on the solvent used and the degree of polymerization (DP). The absorption spectra of $\mathbf{V}$ are nearly identical with or even sharper than those of IV in any solvent used and independent of the solvents. The broadening of absorption spectra in III could not be ascribed to interpolymer association, since the absorbance of III satisfies Beer's law below $5 \times 10^{-5} \mathrm{M}$. This trend is very similar to

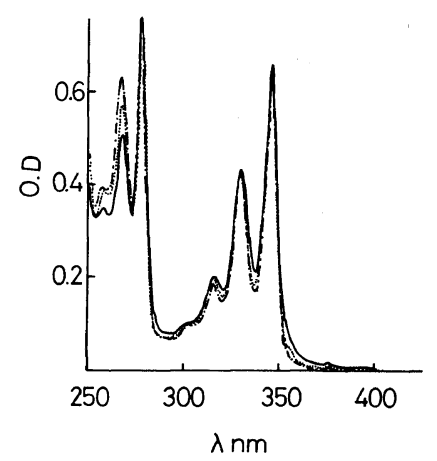

Figure 1. Absorption spectra of III, IV, V, and VI in DCE at room temperature. $[\mathrm{Py}]=2 \times 10^{-5} \mathrm{M}$. III, $(-) ; \mathbf{I V},(----) ; \mathbf{V}, \mathbf{V I},(----)$. the previous results with I. The DP dependent spectral broadening becomes more prominent in DCE than in mesitylene and $o$-xylene. In comparison with $\mathbf{V}$, it is thought that the broadening of absorption spectra in III results from the intrapolymer ground state interaction of $\mathrm{Py}$ with DMA groups. The change in solubility as a function of DP and the resultant change in polymer chain conformation and non-neighboring group participation may possibly be responsible for the solvent and DP dependent ground state interaction.

\section{Shape of Fluorescence Spectra}

The shapes of the fluorescence spectra of III and IV are shown in Figure 2a. It is clear that III and IV display a structureless broad emission around $510 \mathrm{~nm}$ characteristic of the Py*1-DMA exciplex. The exciplex emission is much stronger for III than for IV, whereas the maximum wavelength of the exciplex by IV showed a red shift by $10 \mathrm{~nm}$.

The fluorescence spectra of $\mathbf{V}$ and VI shown in Figure $2 b$ differ very much in the long wavelength region where $\mathbf{V}$ emits emission by the pyrenyl groups at $478 \mathrm{~nm}$. It is apparent that the singlet excited state of pyrene does not interact with phenyl group if the identity of emission by VI is judged with that of the pyrenyl group itself. The emission characteristics of III, IV, V, and VI are shown in Table II.

The excitation profiles of III and IV shown in Figures $3 \mathrm{a}$ and $3 \mathrm{~b}$ reflect the absorption spectra very well. As shown in Figure 3 and Table III, the excitation profiles of III depend considerably on the

Table I. Absorption spectroscopic data of III, IV, and V

\begin{tabular}{|c|c|c|c|c|c|c|c|}
\hline \multirow{2}{*}{ Sample } & \multirow{2}{*}{ Fraction no. } & \multirow{2}{*}{$M_{w}^{\mathrm{a}}$} & \multirow{2}{*}{$M_{w} / M_{n}$} & \multicolumn{4}{|c|}{ DP Broadness of absorption spectrum ${ }^{b}$} \\
\hline & & & & $n$ & DCE & $o$-Xylene & Mesitylene \\
\hline \multirow{5}{*}{ III } & 2 & 22100 & 2.06 & 38 & 0.361 & 0.333 & 0.328 \\
\hline & 4 & 17800 & 1.39 & 30 & 0.357 & 0.332 & 0.324 \\
\hline & 6 & 8900 & 1.24 & 15 & 0.355 & 0.328 & 0.315 \\
\hline & 8 & 6400 & 1.18 & 11 & 0.345 & 0.325 & 0.315 \\
\hline & 10 & 2400 & 1.16 & 4 & 0.331 & 0.322 & 0.315 \\
\hline IV & Monomer model & 507.4 & - & 1 & 0.292 & 0.314 & 0.311 \\
\hline $\mathbf{V}$ & Reference polymer ${ }^{\mathfrak{c}}$ & 10200 & 1.69 & 19 & 0.280 & 0.290 & 0.290 \\
\hline VI & Monomer model of $\mathbf{V}$ & 464.5 & - & 1 & 0.280 & - & - \\
\hline
\end{tabular}

a Relative to standard polystyrene.

b $\mathrm{OD}_{335} / \mathrm{OD}_{346}$ (valley/peak ratio).

c Unfractionated polymer. Molwt estimated by GPC peak elution counts calibrated for standard polystyrene. 
monitoring wavelength, whereas the extent is much less for IV. The excitation spectra for the exciplex emission is broader than that for the monomer
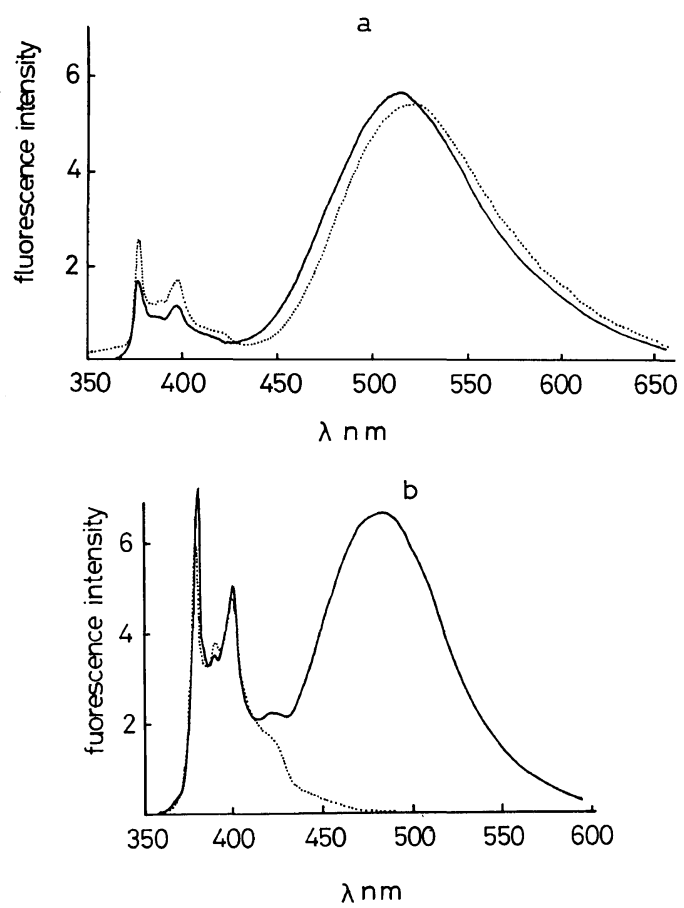

Figure 2. Fluorescence spectra of polymers in DCE. $[\mathrm{Py}]=2 \times 10^{-5} \mathrm{M}$. Excitation at $346 \mathrm{~nm}$. a): III, ( $(-)$; IV, (-----). b): V, (-); VI, (-----). emission as indicated by the valley/peak ratio of the excitation spectra in Table III. Similar results were found in a polyester having pyrenyl and $\mathrm{N}, \mathrm{N}$ dimethylanilino groups in an alternative fashion. ${ }^{2}$
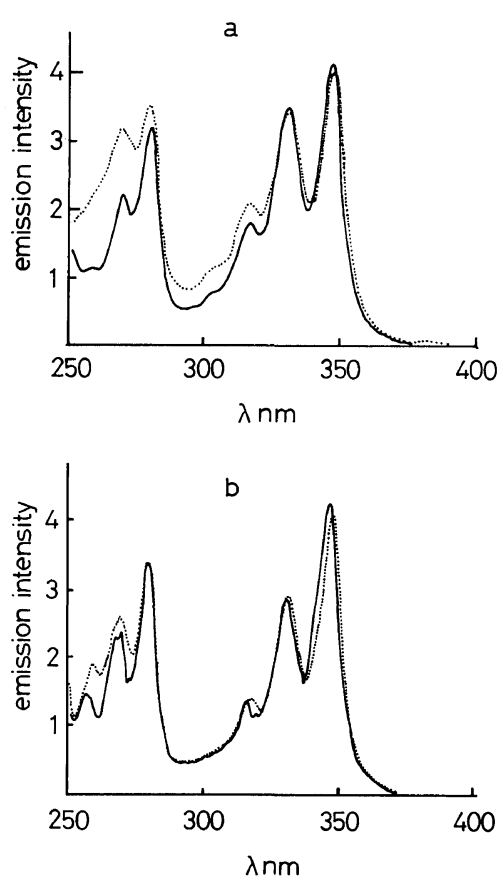

Figure 3. Excitation spectra of polymers in DCE. $\mathrm{OD}^{\max }=0.05$. Wavelength monitored at $377 \mathrm{~nm}(-)$ and $515 \mathrm{~nm}$ (----). a), III (fraction 4); b), IV.

Table II. Emission characteristics of III, IV, and VI

\begin{tabular}{|c|c|c|c|c|}
\hline \multirow{3}{*}{ Sample } & \multirow{3}{*}{ Solvent $^{\mathrm{a}}$} & \multicolumn{3}{|c|}{ Fluorescence spectra } \\
\hline & & $\lambda_{\mathrm{m}}^{\max \mathrm{b}}$ & $\lambda_{\mathrm{e}}^{\operatorname{max~b}}$ & \\
\hline & & $\mathrm{nm}$ & $\mathrm{nm}$ & \\
\hline \multirow{4}{*}{ III-4 } & DCE & 377,398 & 515 & $3.0+1.0$ \\
\hline & $o$-Xylene & $377,386,398$ & 488 & 6.8 \\
\hline & Mesitylene & $377,386,398$ & 485 & 8.0 \\
\hline & DCE & 377,398 & 525 & 2.2 \\
\hline \multirow{2}{*}{ IV } & $o$-Xylene & 377,398 & 493 & 0.60 \\
\hline & Mesitylene & 377,398 & 490 & 0.65 \\
\hline $\mathbf{V}$ & $\mathrm{DCE}$ & 377,398 & $478^{d}$ & $0.98^{\mathrm{d}}$ \\
\hline VI & DCE & 377,398 & - & - \\
\hline
\end{tabular}

${ }^{\text {a }} \varepsilon$ at $25^{\circ} \mathrm{C}, 10.3$ (DCE), 2.57 (o-xylene), and 2.28 (mesitylene). $\eta$ at $25^{\circ} \mathrm{C}$ (cP), 0.73 (DCE), 0.76 ( $o$-xylene), and 0.94 (mesitylene).

b $\lambda_{\mathrm{m}}^{\max }$ and $\lambda_{\mathrm{e}}^{\max }$ are the peaking wavelengths of monomer and exciplex emissions, respectively. $[\mathrm{Py}]=5 \times 10^{-5} \mathrm{M}$.

c Exciplex emission intensity relative to monomer emission intensity extrapolated to zero.

d Excimer emission. No concentration dependence of $F_{\mathrm{e}} / F_{\mathrm{m}}$ was found. 

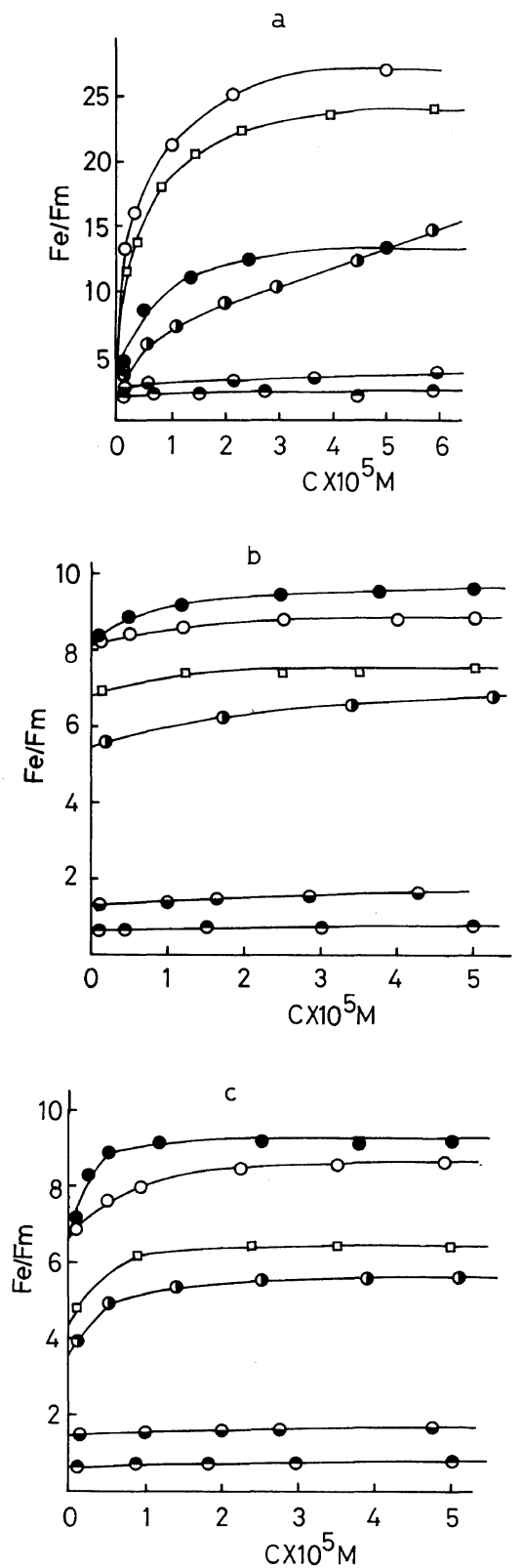

Figure 4. Concentration dependence of exciplex emission by III and IV as a function of DP (excitation at $346 \mathrm{~nm}) \mathrm{a}$ ) in DCE, b) in mesitylene, c) in $o$-xylene: III, Fraction $10(\Theta), 8(\Theta), 6(\square), 4(\bigcirc)$, and $2(O)$; IV, (Ө).

\section{Concentration Dependence of Exciplex Emission}

The concentration dependence of $F_{\mathrm{e}} / F_{\mathrm{m}}$ made possible the separation of intra- and interpolymer
Table III. The valley/peak ratio of excitation spectra of different monitored wavelengths ${ }^{\mathrm{a}}$

\begin{tabular}{|c|c|c|c|c|}
\hline \multirow{2}{*}{$\frac{\begin{array}{c}\text { Monitored } \\
\text { wavelength }\end{array}}{\mathrm{nm}}$} & \multicolumn{2}{|c|}{$F_{337} / F_{346}{ }^{\mathrm{b}}$} & \multicolumn{2}{|c|}{$F_{322} / F_{346}{ }^{\mathrm{b}}$} \\
\hline & III & IV & III & IV \\
\hline 377 & 0.382 & 0.347 & 0.263 & 0.241 \\
\hline 515 & 0.436 & 0.350 & 0.305 & 0.244 \\
\hline Difference $\%$ & +14.1 & +0.8 & +16.0 & +0.12 \\
\hline
\end{tabular}

a Conditions: The maximum OD of sample solution was maintained below $0.05\left([\mathrm{Py}]<10^{-6} \mathrm{M}\right)$.

Solvent, DCE.

b Suffixes indicate excitation wavelength in $\mathrm{mm}$.

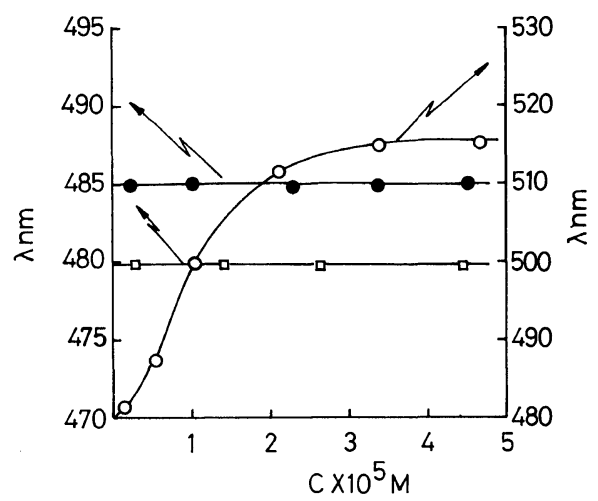

Figure 5. Wavelength of maximum exciplex emission as a function of concentration: III (Fraction 4, molwt, $17800)$ in DCE (O) and in mesitylene (O); IV in DCE ( $\square)$.

exciplexes. The results obtained for III in three solvents are plotted in Figure 4. The strongly concentration dependent exciplex formation in DCE $(\eta=0.73, \varepsilon=10.36)$ as shown in Figure 4a indicates that the contribution of the interpolymer exciplex varies with DP, whereas the contribution of intrapolymer exciplex as given by the $F_{\mathrm{e}} / F_{\mathrm{m}}$ value at infinite dilution is dependent on DP to a lesser extent. On the other hand, the exciplex is formed mostly in an intrapolymer manner in $o$-xylene $(\eta=$ $0.76, \varepsilon=2.57)$ and mesitylene $(\eta=0.94, \varepsilon=2.28)$ as shown in Figures $4 \mathrm{~b}$ and $4 \mathrm{c}$, respectively. The relative intensity of the intrapolymer exciplex emission depends very much on DP suggesting the non-neighboring group participation.

Concentration effects could also be observed on 
the exciplex emission wavelength. The red shift of the exciplex emission peak of III in DCE amounted to $35 \mathrm{~nm}$ with increasing concentration, but such an effect could not be observed for III in mesitylene or $\mathbf{V}$ in DCE as shown in Figure 5.

\section{DISCUSSION}

Ground State Interaction Shown by Broadening of Absorption and Excitation Spectra

The original definition of "exciplex" is a complex formed between an excited molecule and a different kind of molecule in the ground state. Strict differentiation of the exciplex from the excited electron donor acceptor (EDA) complex is however very difficult. Exciplex forming pair bears a more or less donor-acceptor character so that weak pairing in the ground state is likely to occur. The determination of complex formation constant is impossible for a weak interaction system, in particular, under the possible influence of a contact charge transfer complex. A new absorption band is hardly expected to appear and the general trend is a slight broadening of the absorption spectra. Although it is questionable as to whether spectral broadening can be attributed only to charge transnfer interaction, there is no reason for not concluding that certain kind of ground state interactions are present.

This is the general situation when exciplex forming pairs such as aromatic hydrocarbon-amine pairs are concentrated either in molecular aggregates or in solution. The absorption spectra of polymer bound exciplex forming pairs are always broader than the relevant monomer and dimer model compounds. This is also the case with III. Compared with isolated pyrenyl groups in IV, V, and VI, III exhibits the broadest spectrum as judging from the data in Table I. Incidentally, the phenyl groups in $\mathbf{V}$ and $\mathbf{I V}$ are photophysically inert and space filling, thus simulating III and IV, respectively. It is interesting to note that $\mathbf{V}$ and $\mathbf{V I}$ are nearly spectroscopically identical. A high local pyrene concentration does not therefore account for the spectral broadening. In addition, the spectrum of IV is also nearly as sharp as that of VI, indicating that the Py-DMA interaction in a small molecule is weak even if the $n=3$ rule $^{18}$ is satisfied. A comparison of III with I shows that the broadening of I is considerably more than that of III $\left(\mathrm{OD}_{335}\right)$ $\mathrm{OD}_{346}=0.35-0.40$ in DCE, $0.37-0.56$ in mesi- tylene for $\mathbf{I}^{1,2}$ ). Furthermore the broadness of absorption spectra is fairly constant throughout the entire range of DP for III but it increases very much with DP for I. ${ }^{1}$ This is understandable on taking the role of a long main chain spacer in III into account. The Py-DMA pair in III is isolated from other pairs in the polymer so that the energy state is not particularly affected when bonded to the polymer. Consequently, neither is the spectrum of III influenced very much by the change in polymer chain conformation. In contrast to I, the spectra of III are remarkably identical either in a good (DCE) or poor solvent (mesitylene or $o$-xylene). It is thus clear that ground state chromophore interaction occurs. This phenomenon is intrapolymer as far as we could determine by our study and cannot be considered a driving force of interpolymer exciplex formation. On the other hand, the spectral broadening is closely related to the concentration independent (intrapolymer) EDA emission in a glass matrix at $77 \mathrm{~K}$. As a matter of course, the excimer emission by $\mathbf{V}$ is completely lost in the glass matrix and the monomer emission alone is observed as shown in Figure 6. The polymer exhibiting broader absorption spectra in fluid solution shows a stronger EDA emission band centered around $440 \mathrm{~nm}$. The order of the EDA emission intensity is II $>$ I $>$ III, in agreement with the order of the spectral broadening of these polymers. The EDA emission by III or the model compound for IV is very weak; this is consistent with the sharper absorption spectra of monomeric or dimeric model compounds than those of relevant polymers. Additional details of

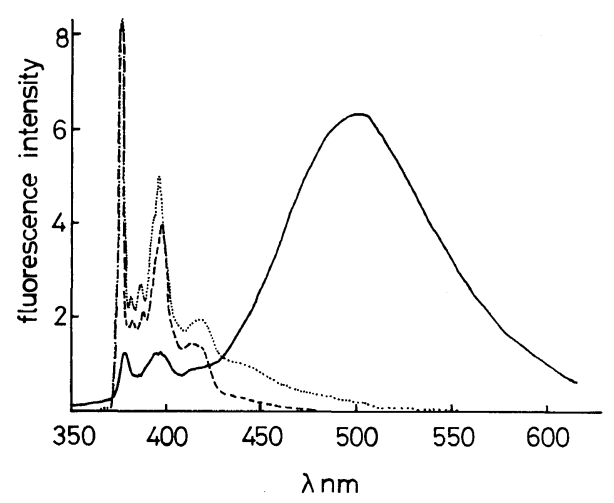

Figure 6. Fluorescence spectra of III and V in MTHF. $[\mathrm{Py}]=5 \times 10^{-5} \mathrm{M}$. Excitation at $346 \mathrm{~nm}$. III at room temp (-), at $77 \mathrm{~K}(-----)$. V at $77 \mathrm{~K}(----)$. 
EDA emission have been published separately. ${ }^{23}$

Strictly speaking, "pure" exciplex formation can be proved only by coincidence of the absorption spectra with the excitation spectra regardless of the wavelength of a monitored emission. This condition is seldom satisfied. In the present systems, the excitation spectra with monitoring emissions at $377 \mathrm{~nm}$ and $515 \mathrm{~nm}$ are different for III, but nearly identical for IV as shown in Table III. The broader excitation spectra with monitoring at $515 \mathrm{~nm}$ is the reflection of the ground state EDA interaction. This is because IV shows little tendency for EDA interaction and consequently the excitation spectra are not sensitive to the monitored wavelength. Although this tendency is comparable with I and II with reference to their model compounds, III exhibits much sharper excitation spectra than I over all monitored wavelength regions in support of the smaller contribution of EDA interaction in III than in $\mathbf{I}$.

These results seems to suggest that the broad structureless emission centered around $500 \mathrm{~nm}$ consists of emission from more than two excited species, the true exciplex emission by definition and perhaps the emission by the EDA complex. Although the general understanding is that EDA and exciplex emissions are not distinguishable, ${ }^{24,25}$ there is evidence that the dynamic behavior of the exciplex and excited EDA complex different as demonstrated for the toluene-1,2,4,5-tetracyanobenzene combination. ${ }^{26}$ If the conversion between the excited state of the EDA complex and exciplex state in the polymer prior to fluorescing requires reorganization of the whole polymer chain, the time constant will not be shorter than the order of $\mu \mathrm{s}-\mathrm{sub} \mathrm{ms}$ which is much longer than the fluorescence lifetime. For example, the intrapolymer terminal group encounter rate constant of polystyrene ranges from $10^{3}$ to $10^{5} \mathrm{~s}^{-1}$ as a function of DP. ${ }^{27}$ This figure is a measure of the chain segment mobility. Consequently, there is a good possibility that the two fluorescing species emit different fluorescence before being stabilized to an identical relaxed state in the polymer system. Our most recent results of fluorescence lifetime measurement confirmed the presence of species corresponding to the excited EDA complex in $\mathbf{I}^{22}$

The contribution of the intrapolymer EDA complex is more important in the high dilution limit where the participation of the interpolymer exciplex
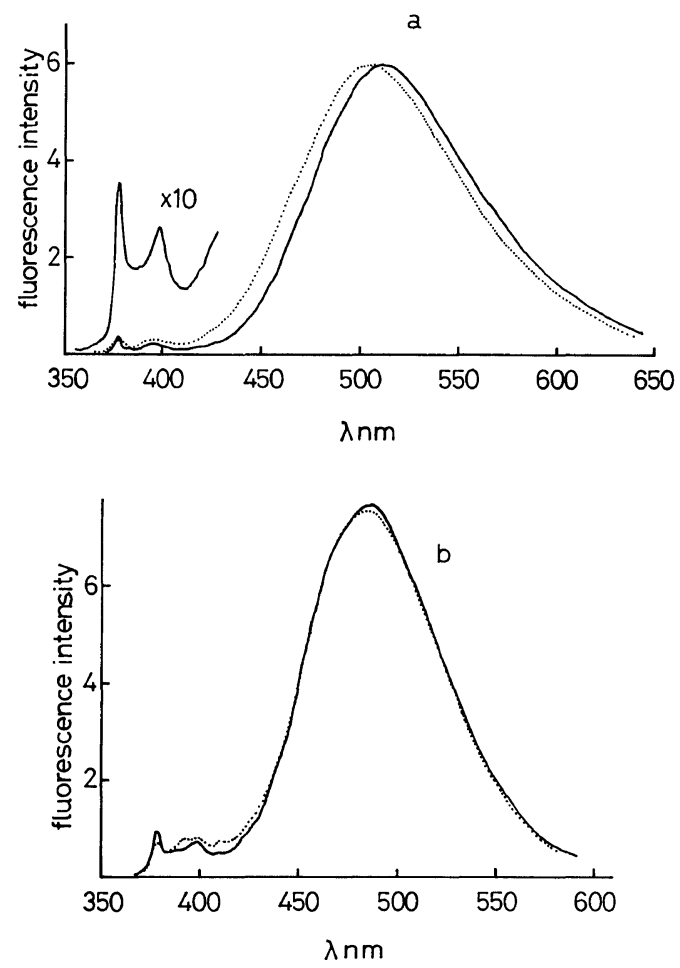

Figure 7. Effects of excitation wavelength on the shape of exciplex emission. III (Fraction 4, mol wt, 17800). a) in DCE, $[\mathrm{Py}]=5 \times 10^{-5} \mathrm{M}$, excitation at $346 \mathrm{~nm}(-)$, and $365 \mathrm{~nm}(-----)$. b) in mesitylene, $[\mathrm{Py}]=4 \times 10^{-5} \mathrm{M}$, excitation at $346 \mathrm{~nm}(-)$, and $365 \mathrm{~nm}(-----)$.

decreases. Since the excitation spectra were measured under an extremely dilute condition $\left([\mathrm{Py}] \ll 1 \times 10^{-5} \mathrm{M}\right)$, the effect of exciting the CT band is more prominently observed than expected from the modest broadening of the absorption spectra. The dependence of excitation spectra on the monitored wavelength is equivalent to the dependence of emission spectra on the excited wavelength in DCE as demonstrated in Figure 7a. The peaking emission wavelength shifts to a lower energy region with increasing the wavelength of excitation. That is, Stoke's shift is smaller for the EDA emission. On the other hand, no shift of peaking wavelength of exciplex emission was detected with the excitation wavelength in a poor solvent (mesitylene) as shown in Figure $7 \mathrm{~b}$ indicating the contribution of the EDA complex is less than in DCE. This trend agrees well with the sharper absorption spectra of III in mesitylene than in DCE. Furthermore, the concen- 
tration independent exciplex wavelength in mesitylene (Figure 5) is consistent with the negligible contribution of the intrapolymer EDA complex. The clear blue shift in DCE with decreasing the concentration of III (Figure 5) may be attributed to the contribution of the short wavelength emission by the EDA complex. Since the separation of "true" exciplex from a small contribution of EDA complex emission is not attainable, we shall call the overall structureless emission an exciplex from hereafter.

\section{Molecular Weight Effects on Exciplex Character- istics}

As reported in previous papers, the characteristics of a polymer becomes more distinctive with increasing mol wt in general. ${ }^{1,2,5,19}$ However, the

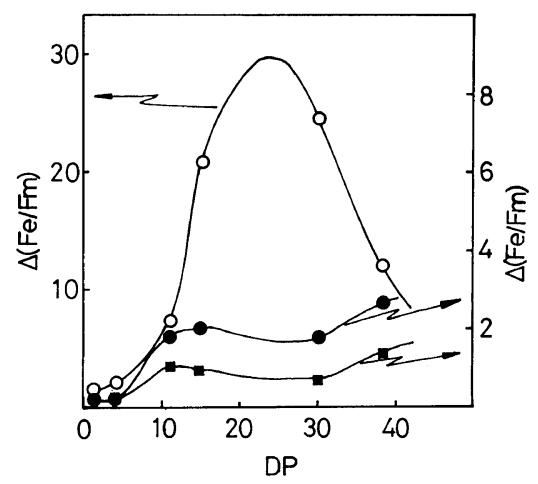

Figure 8. DP dependence of the interpolymer interaction of III in DCE $(\mathrm{O})$, mesitylene $(\square)$, and $o$-xylene (O). $\Delta\left(F_{\mathrm{e}} / F_{\mathrm{m}}\right)=\left(F_{\mathrm{e}} / F_{\mathrm{m}}\right)_{c=2 \times 10^{-s_{\mathrm{M}}}}-\left(F_{\mathrm{e}} / F_{\mathrm{m}}\right)_{c \rightarrow 0}$.

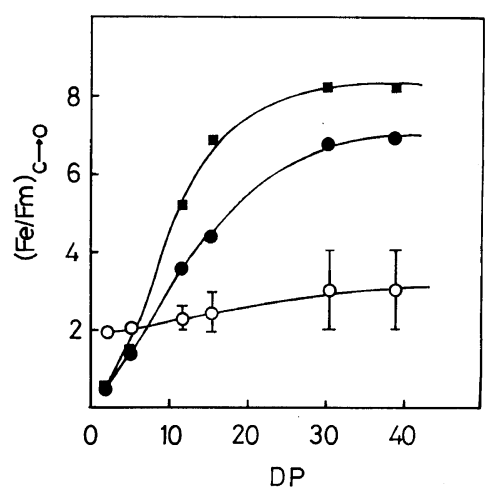

Figure 9. DP dependence of the intrapolymer interaction of III in DCE (O), mesitylene ( $\boldsymbol{\square})$, and $o$-xylene (O). solubility of a polymer chain as a function of $\mathrm{mol} \mathrm{wt}$ and the resultant regulation of chain conformation may provide a different picture. A good example of this is the mol wt dependence of interpolymer association where an optimum mol wt is commonly found. The results in Figure 4 are summarized and depicted in Figures 8 and 9. In a previous publication on $\mathbf{I},{ }^{2}$ the trend of interpolymer association was expressed by the initial slope of the $F_{\mathrm{e}} / F_{\mathrm{m}} v s$. DP plots. As shown in Figure 4, however, the plots for III have no initial linear part so that the initial gain of $F_{\mathrm{e}} / F_{\mathrm{m}}$ at $c=2 \times 10^{-5} \mathrm{M}$ was adopted in Figure 8 instead of the initial slope.

With reference to $\mathbf{I}$, the findings are summarized as follows.

i) The general tendency of III in DCE is very similar to I. In mesitylene and $o$-xylene which are rather poor solvents for this kind of polyester, the DP dependent profile of the $F_{\mathrm{e}} / F_{\mathrm{m}} v s$. $C$ plots are very different from that of $\mathbf{I}$. Nevertheless, the saturation tendency of the $F_{\mathrm{e}} / F_{\mathrm{m}} v s . C$ plots in poor solvents is common to both I and III.

ii) The shape of plots in Figure 8 suggests the presence of two maxima in mesitylene and $o$-xylene. For II, two optimum DP were also observed, one in an oligomer region ${ }^{28}$ and another around $\mathrm{DP}=$ $600-7000^{5,6}$

iii) Intrapolymer exciplex formation by III is nearly independent of DP in DCE but strikingly influenced by DP in the other two solvents. This is the most distinctive difference from $\mathbf{I}$ which exhibits intrapolymer exciplex emission independent of DP in all DCE, mesitylene, and $p$-dioxane. The flexible main chain with a long spacer between the PyDMA pairs in III is favorable for non-neighboring group participation which will certainly be more effective in poor solvents. In good solvents, the extended polymer chains suppress non-neighbouring group interactions and hence the $F_{\mathrm{e}} / F_{\mathrm{m}}$ values at infinite dilution are rather insensitive to DP (Figure 9).

iv) The sharpness of absorption spectra of III (Figure 11) is not affected much by DP compared to that of I (Figure 10 in ref 2). Since the $\mathrm{OD}_{335} /$ $\mathrm{OD}_{346}$ value is an index of EDA complex formation, the results are consistent with the finding of the stronger EDA interaction by I than by III. In addition, the solvent effect on EDA interaction as demonstrated by the excitation wavelength dependence of fluorescence in Figure 7 agrees with the 
broader absorption spectra in DCE. The trend of $\left(F_{\mathrm{e}} / F_{\mathrm{m}}\right)_{c \rightarrow 0}$ as a function of DP in Figure 9 is in contrast to that shown in Figure 11. Since the contribution of EDA emission to $F_{\mathrm{e}} / F_{\mathrm{m}}$ is negligible in $o$-xylene or mesitylene (Figure $7 \mathrm{~b}$ ), the $F_{\mathrm{e}} / F_{\mathrm{m}}$ values are determined exclusively by exciplex emission. The pronounced enhancement of $F_{\mathrm{e}} / F_{\mathrm{m}}$ in poor solvents with increasing DP indicates that the shrinked polymer chain is a favorable condition for intrapolymer exciplex formation. Since the steric requirement of $\mathrm{D}-\mathrm{A}$ arrangement is looser for the exciplex than for the ground state EDA complex, the results in Figure 9 provide an image of randomly gathered D-A pairs in a shrinked polymer chain in poor solvents. In DCE, the polymer chain is extended so that non-neighbouring group participation is weak and $F_{\mathrm{e}} / F_{\mathrm{m}}$ is insensitive to DP as shown in Figure 9.

These results are contrasting to the constant intrapolymer exciplex formation by $\mathbf{I}$ regardless of DP in any of the solvents (Figures 5, 6, and 7 in ref 2). The rigid main chain of $\mathbf{I}$ does not allow nonneighbouring group participation in the relatively low DP region investigated ( $\mathrm{DP} \leq 17)$.

v) The wavelength of the exciplex emission peak is not affected by DP as shown in Figure 10. The measurements were made at a constant concentration so that the relative contributions of intraand interpolymer exciplexes differ from sample to sample owing to DP dependent inter- and intrapolymer exciplex formation as shown in Figures 8 and 9. Consequently, the energy level is the same for the inter- and intrapolymer exciplexes.

\section{Solvent Effects on the Intensity and Wavelength of Exciplex Emission}

The solvent effects on the exciplex emission characteristics given in Table II are as follows. i) Solvent effect on the exciplex wavelength is smaller for III than for IV. The reduced solvent effects in polymer systems are now generally recognized and attributed to the protecting effect and self-solvation by polymer chains. ii) The sequence of $\left(F_{\mathrm{e}} / F_{\mathrm{m}}\right)_{c \rightarrow 0}$ in three solvents is different for III and IV and the value for IV is related to the chain conformation of the trimethylene group whereas $\mathrm{Py}^{* 1}$ in III does not necessarily interact with DMA at the other end of a trimethylene group but encounter DMA at the nonadjacent site. iii) The minor solvent effects on monomer emission by Py*1 are attributed to solvent

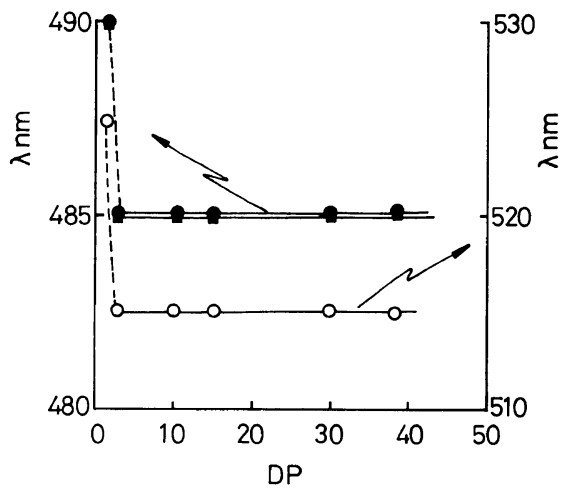

Figure 10. Wavelength of maximum exciplex emission of III as function of DP in DCE (O), mesitylene ( $\square$ ), and o-xylene (O). $[\mathrm{Py}]=5 \times 10^{-5} \mathrm{M}$, excitation at $346 \mathrm{~nm}$.

polarity effects. The vibrational fine structure of Py emission is so sensitive to the solvent polarity that it can be used as a polarity probe. ${ }^{29}$

The choice of solvent was made so as to study the effect of polymer solubility. DCE and $o$-xylene having nearly identical viscosities $(0.73$ and $0.76 \mathrm{cP}$, respectively) were chosen. DCE is a much better solvent than $o$-xylene. The dielectric constant, however, cannot be adjusted. A small difference in the viscosity of $o$-xylene and mesitylene $(0.76$ and 0.94 $\mathrm{cP}$, respectively) is not appreciably reflected on the spectroscopic results. The difference between DCE and $o$-xylene is explicit as shown in Figures 5, 8, and 9. As mentioned above, a shrinked polymer chain in $o$-xylene is unfavorable to interpolymer association (Figure 8) but favorable to intrapolymer exciplex formation (Figure 9). The constant exciplex wavelength in mesitylene over wide ranges of concentration (Figure 5) and DP (Figure 10) indicated that the inter- and intrapolymer exciplexes are not distinguishable from the shape of the emission. In contrasts, the dependence of exciplex wavelenegth on concentration comes to $35 \mathrm{~nm}$ for the high DP sample in DCE. The prominent contribution of EDA complex emission peaking at the shorter wavelength region than true exciplex emission in this solvent (Figures 3a and 7a) accounts for this. By analogy with EDA emission by $\mathbf{I},{ }^{23}$ the formation of the EDA complex by III is favored by high DP polymers. The present interpretation of Figure 5 is not inconsistent with the results in Figure 10. Under the measurement conditions of 
Figure 10, the contribution of EDA emission seems small. In the high DP region, the contribution of the interpolymer exciplex is overwhelming whereas the EDA complex formation is weakened and therefore the intrapolymer exciplex mainly determined the emission wavelength in the very low DP region. The contribution of EDA emission is much less in mesitylene (Figures $3 b$ and $7 b$ ) in support of the concentration independent emission wavelength. One possible reason for the difference between DCE and mesitylene or $o$-xylene is the restricted chain conformation in the poor solvent so that the specific geometry required for the EDA complex formation is not permitted. From photophysical studies of $\alpha$ aryl- $\omega$-( $N, N$-dimethylanilino) alkanes, it is recognized that this strict geometry is not necessary for exciplex. ${ }^{30,31}$ In other words, the enhanced local chromophore concentration may facilitate the exciplex formation but disturb the EDA complex formation. This interpretation also fits the sharper absorption spectra of III in the poor solvents than in DCE (Table I). When the EDA complex formation is favorable, the absorption spectra are broader. These results are different from those of $\mathbf{I},{ }^{2}$ in which the contribution of the intrapolymer exciplex (i.e., $\left.\left(F_{\mathrm{e}} / F_{\mathrm{m}}\right)_{c \rightarrow 0}\right)$ is much smaller and furthermore, independent of DP.

\section{Astonishingly High Quantum Yield of Exciplex Emission in Polymers}

It is of interest to note that the exciplex emission yield of III is very high in a moderately polar solvent (DCE, $\varepsilon=10.4$ ) and furthermore, increases with concentration. The high $\Phi_{\mathrm{e}}$ of 0.40 at $C=$ $2 \times 10^{-5} \mathrm{M}$ is mainly attributed to the interpolymer exciplex formation. In comparison with the intermolecular exciplex formation between $\mathrm{Py}$ and DMA, the value for III is astonishingly high. The $\Phi_{\mathrm{e}}$ for pyrene- $N, N$-dimethylaniline in DCE was reported to be $0.056 .{ }^{32}$ The enhancement of $\Phi_{\mathrm{e}}$ in III indicates that the environment provided by polymer association is very nonpolar and furthermore facilitates the exciplex forming process.

So far as we know, the highest $\Phi_{\mathrm{e}}$ of Py-DMA pair was obtained for 1-(1-pyrenyl)-3-(4- $N, N$ dimethylanilino) propane $\left(\mathbf{P}_{3}\right)$. The compound satisfying the $n=3$ rule allows efficient encounter between Py and DMA and the $\Phi_{\mathrm{e}}$ value reaches 0.40 in $n$-hexane. However, $\mathrm{P}_{3}$ is not protected from solvation and the value decreases very much with

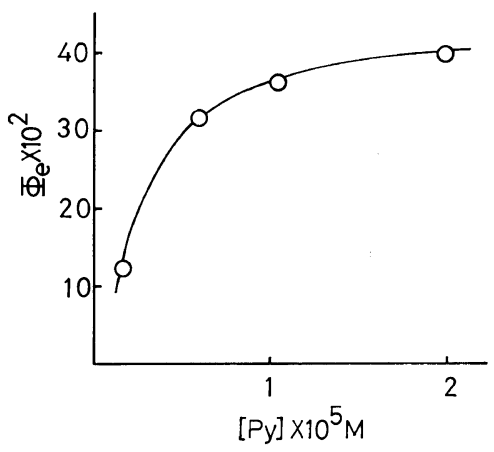

Figure 11. Quantum yield of exciplex emission by III (Fr-4) as a function of concentration in DCE. Excitation at $346 \mathrm{~nm}$ at room temp.

increasing solvent polarity. The $\Phi_{\mathrm{e}}$ is 0.15 in butyl acetate $(\varepsilon=5.29), 0.07$ in pyridine $(\varepsilon=12.4)$, and 0.08 in isobutyl alcohol $(\varepsilon=16.6){ }^{33}$ The value is apparently much smaller than that for III when the macroscopic dielectric constant of the solvent is identical.

\section{CONCLUSION}

The preceding discussions are summarized in Table IV. It is clearly shown that the tendency of the interpolymer association which proceeds smoothly in a good solvent but levels off in a poor solvent is a common trend for I and III. The strong DP dependence of the intrapolymer exciplex/EDA complex indicates non-neighbouring group participation stemming from the flexiable polymer chain connecting the chromophore pairs in III. This is in contrast to $\mathbf{I}$ in which the chromophores are more condensed and the intrapolymer phenomena are independent to DP in either good solvents or poor solvents. The polymer association provides favorable environments for exciplex emission. An $\Phi_{\mathrm{e}}$ of III as high as 0.4 is much larger than that of $I^{22}$ the polyurethane equivalent of $\mathrm{III},{ }^{34}$ or $\mathrm{Py}\left(\mathrm{CH}_{2}\right)_{3}$ DMA. ${ }^{33}$

These intra- and interpolymer phenomena are sensitive to chain rigidity, chromophore concentration and stereochemistry as well as interpolymer binding force. We are now making a study of polyurethane having a comparable structure to III $^{\mathbf{3 4}}$ but capable of hydrogen bonding and the dynamics of exciplex formation in this series of Py-DMA polymers. The polymer structure-molecular inter- 


\section{H. L. YUAN and S. TAZUKE}

Table IV. Comparison of I, II, III, and IV

The mannor in which the polymer structure influences exciplex behaviors.

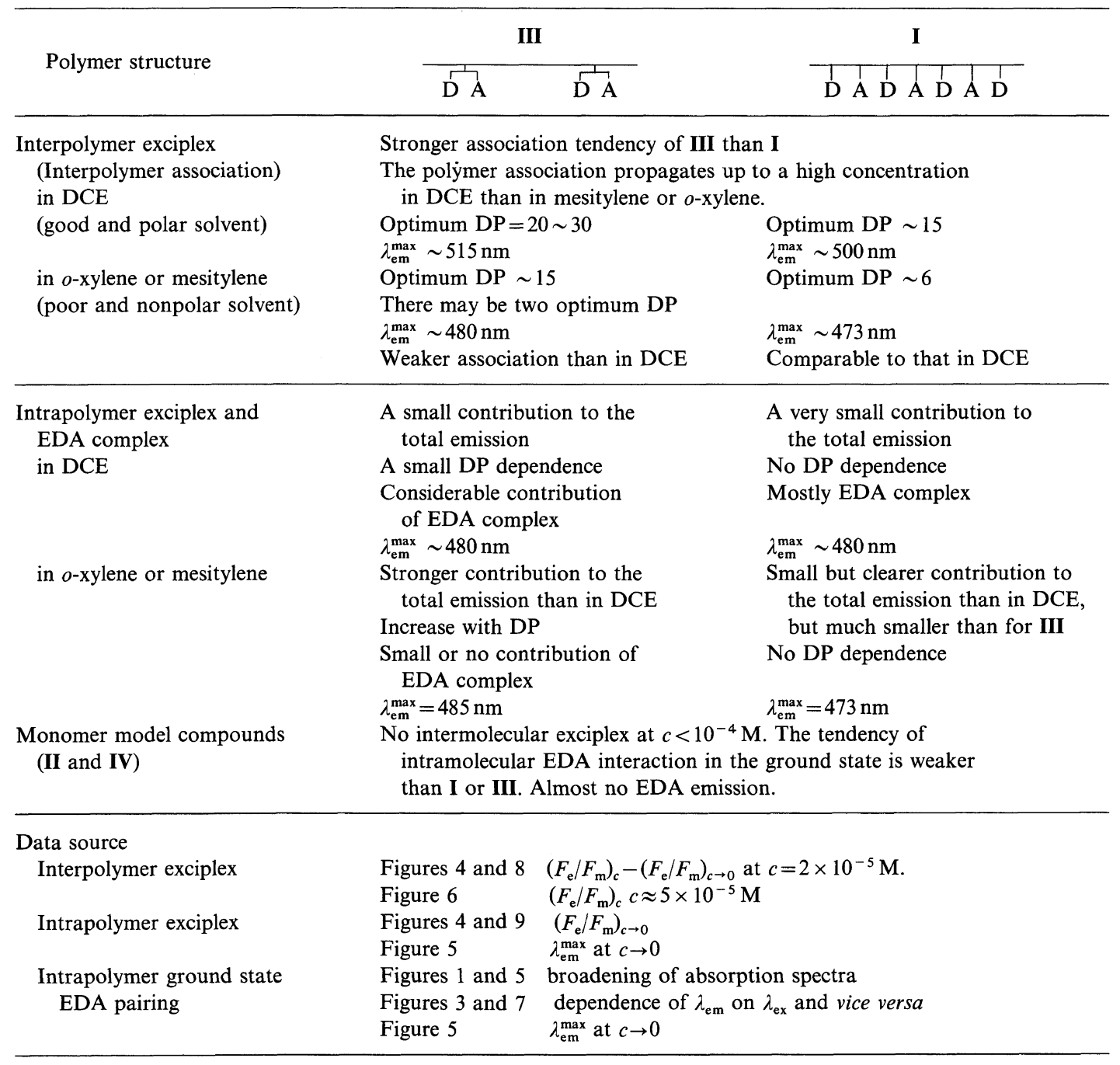

action problem will be further discussed in forthcoming publications.

\section{REFERENCES}

1. S. Tazuke, H. L. Yuan, K. Sato, and Y. Iwaya, Macromolecules, 14, 267 (1981).

2. S. Tazuke and H. L. Yuan, Polym. J., 14, 215 (1982).

3. H. L. Yuan, S. Tazuke, and K. Sato, Polym. Prepr. Jpn., 29, 235 (1980).

4. H. L. Yuan, K. Sato, and S. Tazuke, Polym. Prepr. Jpn., 29, 1329 (1980).

5. Y. Iwaya and S. Tazuke, Macromolecules, 15, 396
(1982).

6. S. Tazuke, Y. Iwaya, and R. Hayashi, Photochem. Photobiol., 35, 621 (1982).

7. S. Tazuke and Y. Matsuyama, Macromolecules, 8 , 280 (1975).

8. Y. Matsuyama and S. Tazuke, Polym. J., 8, 481 (1976).

9. S. Tazuke and Y. Matsuyama, Macromolecules, 10, 215 (1977).

10. S. Tazuke and F. Banba, J. Polym. Sci., Polym. Chem. Ed., 14, 2463 (1976).

11. S. Tazuke and F. Banba, Macromolecules, 9, 451 (1976).

12. S. Tazuke, K. Sato, and F. Banba, Chem. Lett., 1321 
(1975).

13. S. Tazuke, K. Sato, and F. Banba, Macromolecules, 10, 1224 (1977).

14. K. Sato, N. Hayashi, and S. Tazuke, J. Polym. Sci., Polym. Lett. Ed., 15, 671 (1977).

15. S. Tazuke and N. Hayashi, Polym. J., 10, 443 (1978).

16. S. Tazuke and T. Tanabe, Macromolecules, 12, 848 (1979).

17. H. L. Yuan and S. Tazuke, Polym. Prepr. Jpn., 30, 1324 (1981).

18. F. Hirayama, J. Chem. Phys., 42, 3163 (1965).

19. S. Tazuke and H. L. Yuan, J. Phys. Chem., 86, 1250 (1982).

20. K. Miyaki and S. Yamagishi, J. Pharm. Soc. Jpn., 76, 1196 (1956).

21. H. L. Yuan and S. Tazuke, J. Polym. Sci., Polym. Lett. Ed., 20, 81 (1982).

22. S. Tazuke, H. L. Yuan, T. Matsumaru, and Y. Yamaguchi, Chem. Phys. Lett., 92, 81 (1982).

23. S. Tazuke and H. L. Yuan, Polym. J., 14, 695 (1982).

24. M. Itoh and T. Mimura, Chem. Phys. Lett., 24, 551 (1974).
25. E. Gaweda and J. Prochorow, Chem. Phys. Lett., 30, 155 (1975).

26. J. Dresner and J. Prochorow, J. Lumin, 24/25, 539 (1981).

27. W. Schnabel, "Development of Polymer Photochemistry," Vol. 3, N. S. Allen, Ed., Appl. Sci. Publ., London, 1982, p278.

28. R. Hayashi, Y. Iwaya, and S. Tazuke, unpublished data.

29. D. C. Dong and M. A. Winnik, Photochem. Photobiol., 35, 17 (1982).

30. T. Okada, T. Fujita, M. Kubota, S. Masaki, N. Mataga, R. Ide, Y. Sakada, and S. Misumi, Chem. Phys. Lett., 14, 563 (1972).

31. E. A. Chandross and H. T. Thomas, Chem. Phys. Lett., 9, 393 (1971).

32. N. Mataga, T. Okada, and N. Yamamoto, Chem. Phys. Lett., 1, 119 (1967).

33. T. Okada, T. Saito, N. Mataga, Y. Sakata, and S. Misumi, Bull. Chem. Soc. Jpn., 50, 331 (1977).

34. H. L. Yuan and S. Tazuke, Polym. J., 15, 125(1983). 